PART 14.

AGN and Galactic Centre 


\title{
The Dark Mass Concentration at the Galactic Center
}

\author{
F. Melia ${ }^{1}$ \\ Physics and Astronomy Departments, University of Arizona, Tucson, \\ $A Z 85721$
}

\begin{abstract}
Stellar kinematic studies indicate the presence of a concentrated central mass at just under $2 \times 10^{6} M_{\odot}$, in close agreement with the mass deduced from gas velocities measured with the [Ne II] line. Although this mass is most likely a black hole, it may be dominated by a tightly concentrated cluster of stellar remnants. If $\mathrm{Sgr} \mathrm{A*}$, a point radio source coincident with this central mass, is a massive black hole embedded in a region with strong gaseous outflows, as suggested by the observation of $\mathrm{He} \mathrm{I}, \mathrm{Br} \alpha$ and $\mathrm{Br} \gamma$ line emission, it is accreting from its environment via the Bondi-Hoyle process. We discuss the consequences of this activity, including the expected mass and angular momentum accretion rate onto the black hole, and the resulting observable characteristics. The latest infrared images of this region appear to rule out the possibility that this large scale flow settles down into a standard $\alpha$-disk at small radii. We discuss some possible scenarios that might account for this, including strong advection in the disk or the presence of a massive, fossilized disk. Not all of the gas affected in this way by Sgr A*'s strong gravitational field becomes bound. Some of it is redirected into a focused flow that in turn interacts with other coherent gas structures near the black hole. We suggest that the mini-cavity (to the south-west of $\mathrm{Sgr} \mathrm{A}^{*}$ ) may be formed as a result of this activity, and argue that the characteristics of the mini-cavity lend some observational support for the presence of a concentrated mass near Sgr $A^{*}$. We show, however, that as far as the mini-cavity is concerned, this concentrated mass need not be in the form of a point mass, but may instead be a highly concentrated cluster of stellar remnants.
\end{abstract}

\section{Introduction}

The discovery of $\mathrm{Sgr} \mathrm{A}^{*}$ followed a prediction that such an object might be visible at radio wavelengths (Lynden-Bell \& Rees 1971), though the exact mechanism powering the radiative emission has been difficult to identify. Its uniqueness, together with its low proper motion $\left(<40 \mathrm{~km} \mathrm{~s}^{-1}\right.$; Backer \& Sramek 1987) and its location near the dynamical center of the galaxy, suggest that it may be a

\footnotetext{
${ }^{1}$ Presidential Young Investigator
} 
massive point-like object dominating the gravitational potential in the inner 0.5 pc region.

Multi-wavelength observations of the galactic center region have provided a detailed picture of the gas dynamics in the vicinity of $\operatorname{Sgr~A*}$, pointing to the accretion model as perhaps the most likely physical process producing the spectral properties we now attribute to this black hole candidate. Many of Sgr $A^{*}$ 's radiative characteristics may be due to the energy liberated by a galactic center wind as it accretes down the deep potential well. This gaseous flow is inferred to have a velocity $v_{w} \approx 500-700 \mathrm{~km} \mathrm{~s}^{-1}$ and to constitute a mass loss rate $\dot{M}_{w} \approx 3-4 \times 10^{-3} M_{\odot} \mathrm{yr}^{-1}$ (Hall, Kleinmann \& Scoville 1982; Geballe et al. 1991; Serabyn et al. 1991; Yusef-Zadeh \& Melia 1991).

The nature of the Bondi-Hoyle accretion onto a point like object presents somewhat of a challenge in understanding what happens to the gas as it settles down into a planar configuration close to the event horizon. Even if the ambient flow is uniform, fluctuations beyond the bow shock (located at roughly the accretion radius $R_{a c c} \approx 10^{5} R_{g}$, where $R_{g} \equiv 2 G M / c^{2}$ is the Schwarzchild radius) produce a transient accretion of net angular momentum that ought to result in the formation of a temporary (albeit small) disk. More realistically, the inflow itself carries angular momentum, so that the formation of a disk-like structure at small radii (i.e., $r \approx 10^{2-3} R_{g}$ ) is difficult to avoid. In this paper, we will discuss the characteristics expected of the large scale flow should $\mathrm{Sgr} \mathrm{A}^{*}$ be a point-like object with the mass $\left(\sim 1-2 \times 10^{6} M_{\odot}\right.$; Haller et al. 1996) implied by the stellar kinematics, and we will consider several possibilities for the flow at smaller radii.

A related question is whether the gaseous motions near $\mathrm{Sgr} \mathrm{A}^{*}$ are responsible for producing a feature known as the minicavity located in one of the ionized streamers (the Bar) appearing in radio continuum maps to the southwest of the dynamical center (Yusef-Zadeh, Morris \& Ekers 1989). A chain of blobs of emission appearing in more recent, high-resolution radio images seem to lead away from $\mathrm{Sgr} \mathrm{A}^{*}$ toward the cavity, providing some morphological evidence that the two are physically connected. Spectroscopy and $1^{\prime \prime}$ narrow-band imaging of near-infrared line emission in the central $0.3 \mathrm{pc}$ of the Galaxy showed that the mini-cavity is a source of bright $2.2178 \mu \mathrm{m}$ line emission that has been unambiguously identified as [FeIII] (Lutz, Krabbe \& Genzel 1994). This followed earlier detections of $2.217 \mu \mathrm{m}$ emission toward the Sgr A*/IRS 16 region by Eckart et al. (1992), who speculated that the radiation originates within an expanding bubble of ionized, hot iron driven by an outflow from the direction of the massive black hole candidate. This picture is supported by the derived fractional abundances of $[\mathrm{Fe}] /[\mathrm{H}] \geq 2.5 \times 10^{-5}$ and $\left[\mathrm{Fe}^{++}\right] /\left[\mathrm{Fe}^{+}\right] \approx 1$ which, together with the observed spatial distributions and line profiles of [FeIII] and $\mathrm{HI} \mathrm{Br} \gamma$, suggest that an expanding gas bubble is being created in the partially neutral gas streamer by a fast $\left(\sim 1000 \mathrm{~km} \mathrm{~s}^{-1}\right)$ gas flow from one or several sources within a few arcsecs of the present location of the mini-cavity. Lutz et al. (1994) conclude that the most likely physical configuration of the mini-cavity is that of a shocked gas heated and compressed by this interaction, which destroys dust grains and releases iron into the gas phase. The dense postshock gas then forms an HII region, maintained at about $7000 \mathrm{~K}$ electron temperature via photoionization by the central UV field. The idea that dust is being destroyed is 


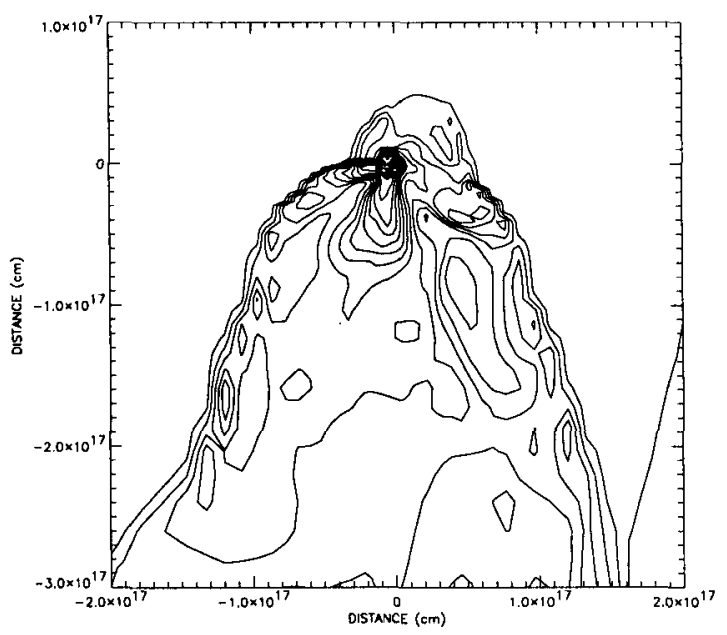

Figure 1. Contour plot of the density $\rho$ in the Bondi-Hoyle bow shock at $\mathrm{Sgr} \mathrm{A}^{*}$ (positioned at 0,0 ). The image has 25 contours increasing as the square root of $\rho$ from $4.8 \times 10^{3}$ to $2.1 \times 10^{5} \mathrm{~cm}^{-3}$. The galactic center wind entering from the top is described in the text and it flows past a black hole with mass $10^{6} M_{\odot}$. The inner boundary is at $0.1 R_{a c c}$.

supported by the finding of Gezari \& Yusef-Zadeh (1991) that the ratio of $12 \mu \mathrm{m}$ dust continuum to radio continuum flux has a minimum at the position of the mini-cavity.

In addition to considering the large scale infall onto the mass concentration near Sgr A*, we shall also address the issue of whether the "excess", highly focused, hydrodynamic stream past $\mathrm{Sgr}^{*} \mathrm{~A}^{*}$ has the correct distribution to be consistent with the gaseous flow required in the detailed modeling of the structure of the mini-cavity (Lutz et al. 1994). Knowing the IRS16 wind characteristics and $\mathrm{Sgr} \mathrm{A}^{*}$ 's mass provides an unambiguous profile of the collimated post-bow shock wind. We shall examine this situation both in the case where the mass concentration is in the form of a point-like object and where it is represented by a highly condensed cluster of stellar remnants. The latter may be of potential interest to an alternative scenario for the activity in this region, in which the concentrated mass may be in the form of a collapsed core in a cluster of white dwarfs (see, e.g., Haller et al. 1996). Although we make no attempt here to justify such a distribution on dynamical and evolutionary grounds, we do nontheless explore its viability in accounting for large scale features such as the minicavity. In this picture in which no massive black hole is present in the region, $\mathrm{Sgr} \mathrm{A}^{*}$ might then simply be the hot, magnetized gas trapped in the core of the stellar distribution, not unlike the X-ray emitting plasma in the potential well of clusters of galaxies (Melia \& Haller 1996). 


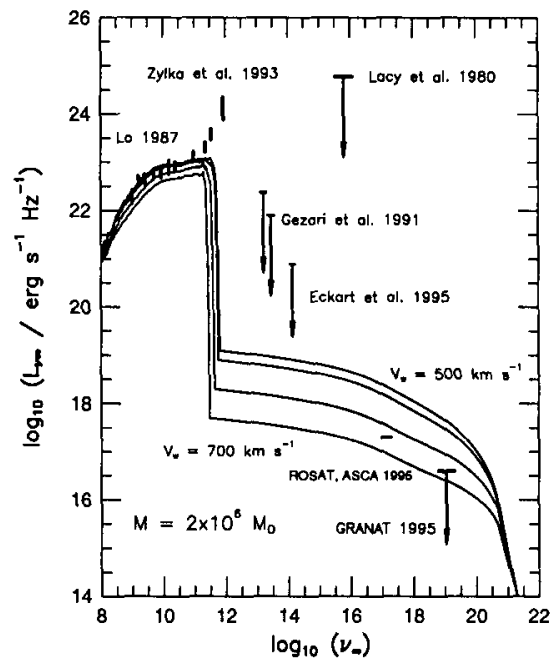

Figure 2. Bremsstrahlung and Magnetic Bremsstrahlung spectrum from the post bow-shock infall toward the black hole. The various curves range from a wind velocity of $500 \mathrm{~km} \mathrm{~s}^{-1}$ (upper) to $700 \mathrm{~km} \mathrm{~s}^{-1}$ (lower).

\section{Bondi-Hoyle Accretion Onto Sgr $A^{*}$ and Radiative Emission}

A characteristic length scale $\left(R_{a c c}\right)$ for accretion onto a compact object such as $\operatorname{Sgr} A^{*}$ is given by the condition that the potential energy density of the flowing gas is matched to its kinetic energy density. In Figure 1 we show the cross-sectional density contours resulting from a 3-D hydrodynamic simulation with a domain of solution spanning a region of $30 R_{a c c}$ in either direction, using the black hole and galactic center wind characteristics discussed in $\S 1$ above (see also Ruffert \& Melia 1994; Melia, Coker \& Yusef-Zadeh 1996; Coker \& Melia 1996). Compression of the gas and magnetic field dissipation in the converging flow toward the black hole heat the gas, which in turn cools primarily by bremsstrahlung and magnetic bremsstrahlung emission with an emissivity characteristic of the particle density $n$, temperature $T$ and magnetic field intensity (Melia 1994). For the conditions in the galactic center, $n$ scales roughly as $r^{-3 / 2}$ with a value of about $2 \times 10^{11} \mathrm{~cm}^{-3}$ at $r=R_{g}$, and $T$ varies from about $10^{6} \mathrm{~K}$ behind the shock to as high as $\sim 10^{10} \mathrm{~K}$ in the very inner region. Figure 2 shows the broad-band spectrum expected from this post-shock flow, in which the emissivity shortward of $\sim 10^{12} \mathrm{~Hz}$ is due primarily to thermal cyclotron/synchrotron radiation, whereas the flux at higher energies results from bremsstrahlung processes. In this figure, the ROSAT data point is consistent with the ASCA measurement when the $N_{H} \approx 2.2 \times 10^{23} \mathrm{~cm}^{-2}$ of column density in the accreting flow is taken into account in estimating the line-of-sight absorption. Since the soft X-rays are produced gradually as the gas descends toward the event horizon, the overlying column density in the Bondi-Hoyle flow adds to 


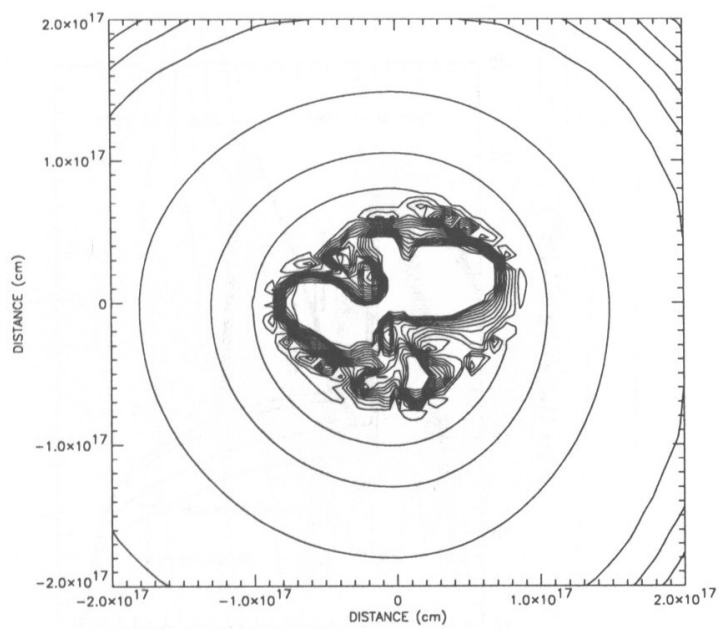

Figure 3. Cross-sectional contour map of the mechanical energy flux flowing into the minicavity. The image shows 20 contours increasing as the square root of the energy flux from 1,100 to $16,500 \mathrm{ergs} \mathrm{cm}^{-2}$ $\mathrm{s}^{-1}$. Note that the inner portion is low in flux due to the removal of the gas by accretion onto the black hole.

the overall $N_{H}$ associated with this emission. We shall return to the question of what happens to this gas when it approaches the black hole in $\S 4$.

\section{Formation of the Mini-Cavity}

In Figure 3, we show a cross-sectional contour map of the kinetic energy flux carried by the focused "excess" flow behind the accretor, at the observed location of the minicavity. The radius of the post-bow shock flow corresponds very closely with the observed radius ( $\sim 1^{\prime \prime}$, see Yusef-Zadeh et al. 1990) of the mini-cavity, suggesting that the energy required to dynamically control the activity in this hole is brought in by the mechanical flux. Indeed, Lutz et al. (1993) conclude in their detailed analysis of the cavity's emission that the power required to sustain its luminosity is $L_{c a v} \approx 6 \times 10^{37} \mathrm{ergs} \mathrm{s}^{-1}$ for reasonable values of the surrounding medium parameters. Our calculated value of the collimated flow's mechanical luminosity $\left(L_{\text {shell }} \approx 2.5 L_{\text {cav }}\right.$ ) is therefore about right to be the dominant energizing agent (Melia, Coker \& Yusef-Zadeh 1996). We note that if the black hole mass is larger than the $\approx$ one million solar masses assumed here, then the focusing power of the accretor is greater and we would expect a larger mechanical luminosity and mass flux into the streamer. In addition, since the accretion radius scales as the mass, we would then also expect a larger size for the mini-cavity. 


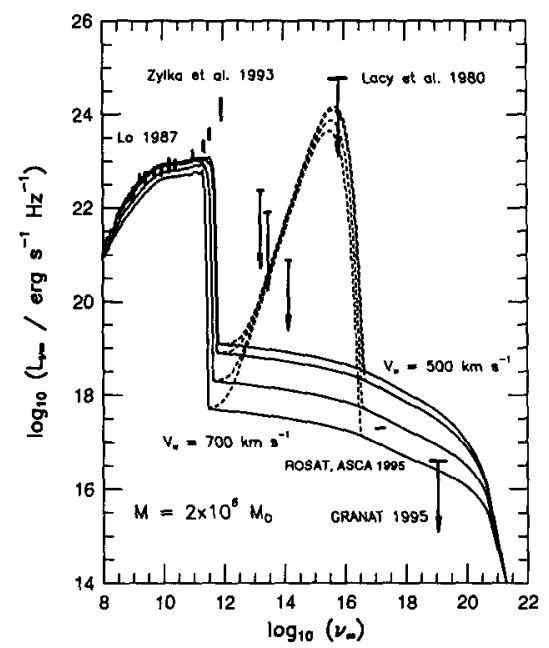

Figure 4. Same as Fig. 2, but now with the additional component (dotted curves) due to an $\alpha$-disk forming at smaller radii. The disk size and accretion rate are determined by the mass and angular momentum inflow rates from the Bondi-Hoyle shock.

\section{Accretion Onto the Black Hole}

Looking at the density profile in Figure 1, we would infer that even when the upwind medium is uniform, the downwind region exhibits fluctuations in $\dot{M}$ that carry a net angular momentum down to the black hole. Detailed simulations (e.g., Ruffert \& Melia 1994) suggest that at any given time, the accreting gas should circularize between 5 and $10 R_{g}$. If the upwind medium has gradients in $\rho$ or $v_{w}$, as might arise from a distributed wind source, this circularization radius might be even bigger. We have calculated the spectral component expected from a sum of blackbody emissions due to this disk (under the assumption that it is a standard $\alpha$ model), and show it as dotted curves in Figure 4. Since the infrared data are seemingly inconsistent with this additional spectral component, we conclude that either (1) the circularized flow does not form an $\alpha$-disk, but rather advects most of its dissipated energy through the event horizon (Narayan \& Mahadevan 1995), (2) the Bondi-Hoyle flow merges into a massive, fossilized disk, storing most of the deposited matter at large radii (Falcke \& Melia 1996), or (3) Sgr A* is not a point-like object (see below).

The first of these possibilities seems very promising. Every disk is advective to some degree. The question is whether the conditions in and around Sgr A* will permit a self-consistent disk solution with a very large advected fraction when the essential physics, including non-local effects and non-Keplerian flows, is incorporated into the analysis. In the second scenario, the wind infall must have a very large specific angular momentum, for it will otherwise settle onto the disk at small radii, where its kinetic energy will be thermalized and radiated away. A fossilized disk would thus need to function as a mass storage device, 


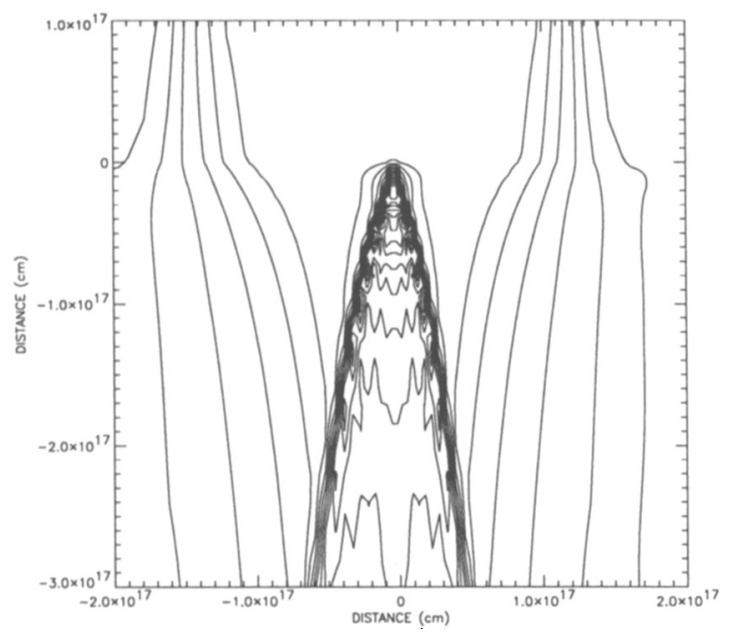

Figure 5. Gas condensation resulting from a Galactic center wind flowing past a cluster of $10^{6}$ one solar mass stellar remnants concentrated within a radius of $2 R_{a c c}$ at the location of $\mathrm{Sgr} \mathrm{A}^{*}$. The image shows 25 contours scaling as the square root of the density from $1.3 \times 10^{2}$ to $7 \times 10^{4} \mathrm{~cm}^{-3}$.

undergoing episodic accretion instabilities, not unlike those observed in X-ray Novae. The third possibility is discussed in the next section.

\section{A Compact Cluster of Stellar Remnants ?}

Perhaps as many as $2-3$ million white dwarfs could have formed and settled within the central cluster (Haller et al. 1996). Whether this distribution of stellar remnants could have undergone core collapse, however, is still not known. The reason this is of potential interest to our understanding of $\mathrm{Sgr} \mathrm{A}^{*}$ is that a concentration of $1-2$ million solar masses within $1-2 R_{a c c}$ could in principle mimic the environmental impact of a point object. In that case, $\mathrm{Sgr} \mathrm{A}^{*}$ itself might simply be the trapped hot, magnetized plasma within the cluster (Melia \& Haller 1996). We have carried out several 3D hydrodynamic simulations of a galactic center wind flowing through such a condensed (isothermal) cluster of one solar mass objects and the density profile of one of these is shown in Figure 5. Of course, unless the enclosed mass is $M_{\text {cluster }}=2 G / v_{w}^{2} R_{\text {cluster }}$, no shock will form since $R_{\text {cluster }}$ will then exceed $R_{a c c}$. The case shown in Figure 5 is for $R_{\text {cluster }}=2 R_{\text {acc }}$. We see, however, that even in this case a condensation of the mass flow past the central mass has several features in common with the downwind Bondi-Hoyle flow we saw in Figure 1. When we examine the size and luminosity of the cavity produced in the Bar due to this type of flow (see Figure 6), the expected characteristics are not so different that we can immediately rule this picture out. To be sure, the minicavity should then be, smaller and the luminosity is about half of the value we inferred in Figure 3. 


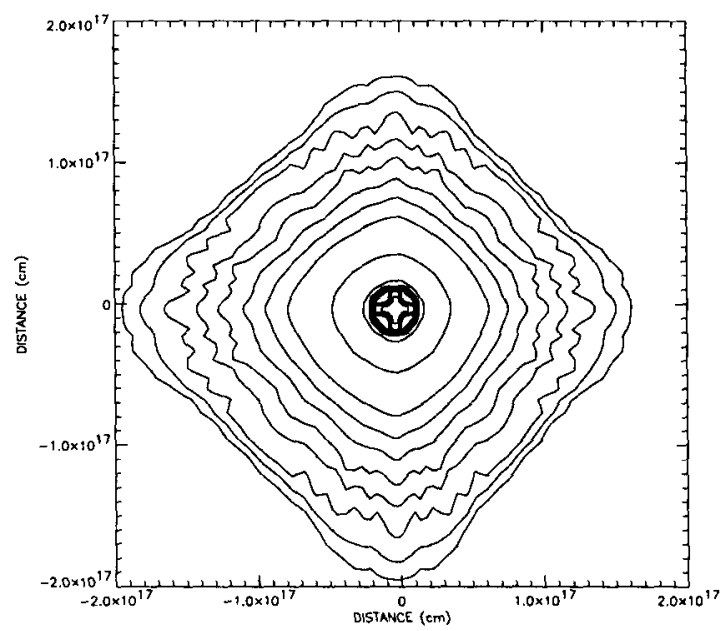

Figure 6. Cross-sectional contour map of the mechanical energy flux flowing into the minicavity for the case shown in Fig. 6. The image shows 20 contours increasing as the square root of the energy flux from 23 to 8,190 ergs $\mathrm{cm}^{-2} \mathrm{~s}^{-1}$.

Nonetheless, this scenario is sufficiently intriguing that it merits further study. The most important test will be to see if the trapped gas can in fact account for the observed radio (and possibly $\mathrm{X}$-ray) spectrum from this source.

\section{Conclusions}

While the nature of the mass concentration in the inner $\sim 0.5 \mathrm{pc}$ of the galaxy is still not quite fully understood, we may already begin to explore the effects of its interaction with the nearby gaseous environment. We have seen that whether or not the mass is point-like, the gravitational focusing of the surrounding gas can lead to a directed flow whose characteristics are those required to produce the mini-cavity to the southwest of $\mathrm{Sgr} \mathrm{A}^{*}$ 's position. In this regard, the hydrodynamical simulations carried out to date support the conclusions regarding the dark mass in this region derived from a study of the stellar kinematics. If Sgr $\mathrm{A}^{*}$ is a massive black hole, its radio emission may be due to the energy liberated by the accreting gas as it descends down into the potential well. However, this matter probably does not flow directly through the event horizon, and one must therefore account for the dissipated Keplerian energy of the gas as it settles into a planar inflow at smaller radii. The observed critically low infrared flux from this source may be an indication that most of this orbital energy is advected inwards, rather than radiated locally as in an $\alpha$-disk. If the central mass concentration is a cluster of stellar remnants, Sgr A* may the radio emission associated with the trapped, hot, magnetized gas in the shallow potential well of the cluster. This possibility is the subject of ongoing work. 
Acknowledgments. This work was supported by NSF grant PHY 88-57218 and NASA grant NAGW-2822.

\section{References}

Backer, D.B. \& Sramek, R.A. 1987, in AIP Proc. 155, ed. D.B. Backer, 155

Coker, R.F. \& Melia, F. 1996, these proceedings.

Eckart, A., Genzel, R., et al. 1992, Nature, 355, 526

Eckart, A., et al. 1996, these proceedings

Falcke, H. \& Melia, F. 1996, ApJ, submitted

Gezari, D. \& Yusef-Zadeh, F. 1991, in Astrophysics with Infrared Arrays, ed. R. Elston, A. S. P. Conference Series Vol. 14, p. 214

Hall, D.N.B., Kleinmann, S.G. and Scoville, N.Z. 1982, ApJ, 260, L63

Haller, J.W., Rieke, M.J., Rieke, G.H., Tamblyn, P., Close, L. \& Melia, F., 1996, ApJ, 456, 194

Lo, K.Y. 1987, in AIP Proc. 155, ed. D.C. Backer, (AIP: New York), 155, 30

Lutz, D., Krabbe, A. \& Genzel, R. 1993, ApJ, 418, 244

Lynden-Bell, D. \& Rees, M. 1971, MNRAS, 152, 461

Melia, F. 1994, ApJ, 426, 577

Melia, F., Coker, R.F. \& Yusef-Zadeh, F. 1996, ApJ, 460, L33

Melia, F. \& Haller, J.W. 1996, ApJ, in preparation

Narayan, R. \& Mahadevan, R. 1995, Nature, 374, 623

Ruffert, M. \& Melia, F. 1994, A.A., 288, L29

Serabyn, E., Lacy, J.H., \& Actermann, J.M. 1991, ApJ, 378, 557

Yusef-Zadeh, F. \& Melia, F. 1991, ApJ, 385, L41

Yusef-Zadeh, F., Morris, M. \& Ekers, R.D. 1990, Nature, 348, 45

\section{Discussion}

W. Duschl: Not a question, but a comment. More recent sub-mm measurements confirm our earlier reported observation that the $\sim 10^{12} \mathrm{~Hz}$ spectrum in Figure 2 is rising.

F. Melia: That's a useful diagnostic since it may signal the presence of a separate spectral component, such as might arise from synchrotron emission near a shock at small radii.

S. Chakrabarti: I believe that the accretion rate in this source is really small ( $~$ $10^{-6} M_{\odot} \mathrm{yr}^{-1}$ ) since the stars have to be kept in their realistic 3D locations. The accretion rate would go down due to the non-cancellation of angular momentum.

F. Melia: That might actually present a more challenging problem since a higher specific angular momentum would then lead to a larger accretion disk. The issue of hiding the infrared emission from such a structure becomes even more accute. However, what you say is indeed at least partially true. Our more recent 
simulations, with a distribution of point wind sources rather than a continuous inflow, do show that since a complete bow shock does not form around $\mathrm{Sgr} \mathrm{A}^{*}$, the flow velocity past the accretor is higher, resulting in a smaller capture rate for the gas.

S. Chakrabarti: If viscosity is really small, then the energy is almost constant (not radiating) and the flow does not have to be "advection dominated" (in which a large amount of energy is generated but not radiated). It can just be a constant solution from Chakrabarti (1989).

F. Melia: Well, actually the viscosity is not the relevant factor here. What matters is the size of the disk. The dissipated power depends on the rate at which matter moves into the potential well, so the disk luminosity is a function of the geometry rather than the viscosity.

S. Chakrabarti: I am more comfortable with a circularization radius of $\sim 50-$ $100 r_{g}$ (and not a million or more, which the advection model of Narayan et al. suggests), since my solutions of viscous transonic flow typically are Keplerian until $\sim 50-100 r_{g}$. 